\title{
Correlated Drug Action as a Baseline Additivity Model for Combination Cancer Therapy in Patient Cohorts and Cell Cultures
}

\author{
Adith S. Arun ${ }^{1,2}$, Sung-Cheol Kim ${ }^{1,3}$, Mehmet Eren Ahsen ${ }^{4}$, Gustavo Stolovitzky1, $\dagger$ \\ 1 IBM T. J. Watson Research Center, Yorktown Heights, New York 10598, USA \\ 2 Department of Applied Mathematics and Statistics, Johns Hopkins University, Baltimore, Maryland \\ 21218, USA \\ 3 Psychogenics, Inc, Paramus, New Jersey, 07652, USA \\ ${ }^{4}$ Gies College of Business, University of Illinois at Urbana-Champaign, Illinois 61820, USA
}

$\dagger$ To whom correspondence should be addressed: gustavo.stolo@gmail.com

\begin{abstract}
Identifying and characterizing the effect of combination cancer therapies is of paramount importance in cancer research. The benefit of a combination can originate in the inherent heterogeneity in the response across patients, the heterogeneity in the clonal composition of tumors in each patient, or in molecular synergy between the compounds given in combination. The effect of drug combinations is usually studied in cell culture, in patient populations or both. To shed light and help characterize combinations and their enhanced benefits over single therapies, we introduce Correlated Drug Action (CDA) as a baseline additivity model. We formulate the CDA theory and propose to model it using a closed-form expression both in the temporal domain (tCDA) to explain survival curves, and in the dose domain (dCDA), to explain dose-response curves. CDA can be used in clinical trials and cell culture experiments. At the level of clinical trials, we demonstrate tCDA's utility in identifying non-additive combinations, and cases where the combination can be explained in terms of the monotherapies. At the level of cells in culture, dCDA naturally embeds null models such as Bliss additivity, the Highest Single Agent model, the dose equivalence principle and sham combinations. We demonstrate the applicability of dCDA in assessing non-additive combinations in experimental data by introducing a new metric, the Excess over CDA (EOCDA). CDA is a general framework to model additivity at the cell line and patient population levels and can be used to characterize and quantify the degree of non-additivity in drug combination therapy.
\end{abstract}

Keywords: Combination Cancer Therapy, Additive Models, Synergistic Drug Combinations

\section{Introduction}

Combination therapy, the principle of treating a patient with multiple drugs either simultaneously or in succession, is a widely used strategy for treating conditions ranging from HIV/AIDS to cancer [1-4]. 
In the 1960's, Frei and Freirich reasoned that cancer cells could mutate and become resistant to one chemotherapy but were less likely to become resistant to multiple different anti-cancer chemotherapy agents [5]. Since that pioneering work, combination therapies have become the de facto approach for treating cancer. However, predicting and quantifying the effects of combination therapies can be experimentally inefficient given the number of possible combinations and resources such as participants, physicians, and assays needed. Thus, it is important to develop models that can characterize the effects of drugs acting in combination. Additive models, that is, models that assume the combination effect results from some sort of superposition of the individual effects of the monotherapies, can serve as a baseline for the expected effects of drugs applied in combination [6]. For example, additive models can be used as a reference to determine if a combination is synergistic.

The effect of combinations have been studied at two fundamentally different levels: cultured cells (or in-vitro) and living organisms (or in-vivo). Cell lines and organoids fit into the former category whereas Patient Derived Xenografts (PDXs) and human clinical trials are examples of the latter. At the culturedcell level, research typically focuses on the dose response at a fixed time point after drug administration; at the living-organism level, research tends to focus on survival time at fixed doses. We will refer to additive models at the level of cultured cells as dose-space models since the primary variable to change is the dose of the monotherapies in the combination. Similarly, models describing patient populations will be referred to as temporal models given that the main endpoint is survival times.

Recently, Palmer and Sorger [7] proposed to describe additive effects of drug combinations in survival analysis in patient cohorts using a framework based on the principle of Independent Drug Action (IDA). IDA is a temporal model that in its most basic form postulates independence between the individual monotherapies that constitute the combination and posits that the survival of the patient treated with a combination of drugs is equal to the survival that the patient would have had if they had been treated with the monotherapy with larger survival time. This independence-based additive model can be useful to model combinations in patients under the assumption that the mode of action of one of the drugs does not affect the mode of action of the other drug. Palmer and Sorger's work clearly articulated that the response to drug combinations in a patient population could be attributed, at least in part, to the response heterogeneity in the treated population. The IDA model, therefore, brought patient-to-patient heterogeneity to the forefront of additivity models in the temporal space.

One of the essential features in Palmer and Sorger's work is the assumption that, even though drugs in a combination are assumed to act independently, the response of two drugs in the same patient could be correlated. Because of the essential role played by this correlation in the subsequent analyses, we believe that it may be more appropriate to call the resulting framework Correlated Drug Action (CDA). In the context of studying the survival times in patient populations as in [7] we will call the framework the temporal CDA or tCDA. In tCDA the monotherapies in a combination may be correlated through confounding factors even if they do not causally affect each other. The nature of this correlation is difficult to ascertain in general, and many models can be developed that result in a given correlation between the outcomes of different monotherapies. We will show that the differences between different 
correlated models is small and in this paper we chose one type of correlated model that allows us to formulate a closed-form and interpretable expression for the effect of the combinations in terms of the effects of the monotherapies (See Supplement). We also provide a statistical framework to decide whether or not we are justified to discard the tCDA model in favor of alternative explanations.

Extrapolating the temporal ideas about correlations between survival times of each patient in a cohort under each monotherapy to the dose-space, we propose the dose CDA or dCDA model that describes the effect of combinations in cell cultures in terms of the dosages of each monotherapy that kill each cell in culture after a given treatment time. Additive models at the level of cell cultures are much more developed [8-10] than those describing survival times of combinations in patient populations [11]. Amongst the most-used reference dose-space additive models are Bliss additivity [12], Loewe additivity [13], the Dose Equivalence Principle [14], and Highest Single Agent (HSA) [15]. Our formulation of the dCDA model naturally embeds Bliss additivity and the HSA model, while incorporating the dose equivalence principle and the requirement of compliance with sham combinations.

Both the temporal and dose-space CDA models are based on the assumption that neither drug directly affects the other. Broadly, non-interaction means that the two monotherapies do not affect one another at the cellular, tissue, organ, or organism level. For the temporal model, tCDA allows for there to be a correlation between the survival times under either monotherapy for each patient. This correlation cannot be directly measured, and can only be inferred in terms of the effects it produces in a population. Likewise, for the dose-space model, the dCDA accounts for a correlation between the lethal doses under either monotherapy for each cell in a cultured population of cells. The range of correlation values $([-1,1])$ allows for a gamut of possible outcomes for the effect of a combination under dCDA. For the dose-space CDA, our model describes a model closer to Bliss additivity (when correlation is close to 0 ) or sham-combination-compliant HSA (when correlation is closer to 1 ). We must make clear that the correlation described in either model cannot be directly measured experimentally because it is not possible to treat a given cell with one monotherapy and then reset to the pre-initial monotherapy conditions and administer the other monotherapy. Thus, the correlation is a latent variable that must be estimated from the data.

We need not constrain ourselves to combinations of only two drugs. By extending the definition of monotherapy to include any reported outcome from a set of therapies, the CDA framework allows for a combination to be composed of more than two drugs, say $A, B$, and $C$, so long as there is data available for drug $C$ and for drug $A$ and $B$ together. Thus, the term monotherapy can be applied liberally and need not only represent a single drug. In many of the clinical trials analyzed in this work, there are three or four drugs that form the combination as such an approach has been historically successful [16-19].

We apply our tCDA null additivity model to public oncology clinical trial data in order to demonstrate its ability to explain the effect of clinical combination therapies and test for non-additive interactions at work within these combinations. Furthermore, we estimate, as a parameter in the model, the correlation of the combination which has potential biological implications. The correlation can potentially inform 
whether there may exist a biomarker or patient covariate that can decouple the patient population into sub-populations that can benefit more from one monotherapies over the other, or those that realize the benefit of the combination.

Rather than introducing an entirely new model in an area that has seen much debate [20], the dosespace CDA null additivity model seamlessly interpolates different individual concepts (Bliss, HSA, the dose equivalence principle and sham combination compliance) and creates a spectrum of non-interaction possibilities between these established models. This semi-unification occurs rather naturally from the basic premises of the approach used to develop the CDA model. We show that the dose-space CDA model is useful in assessing whether there are non-additive effects in a given combination tested on cultured cells. Additionally, we introduce a new performance metric we call Excess over CDA (EOCDA), and compare it to the commonly used Excess over Bliss (EOB) metric in the tested combinations.

\section{Results}

\section{Temporal Correlated Drug Action as a null additive model for understanding combina- tion therapy in human clinical trials}

As a representative example of the tCDA model, we can examine the combination of chemotherapy and Trastuzumab (Herceptin). Chemotherapy consisted of anthracycline plus cyclophosphamide for patients who had never received anthracycline before or paclitaxel for patients who had received adjuvant anthracycline [21]. This therapy is a current treatment for HER-2 overexpressing breast cancers. The Tratuzumab and chemotherapy combination shows a clinical benefit over either individual monotherapy with respect to progression-free survival (PFS) time (Fig. 1A). At around 12 months, the benefit of the combination reduces to that of the Trastuzumab treated group. The tCDA model predicts the PFS curve $S_{A B}(t)$ of the combination of the two drugs A and B (in this case chemotherapy and Trastuzumab) based on the PFS curves of each of the monotherapies $S_{A}(t)$ and $S_{B}(t)$ in the form prescribed by the mathematical expression

$$
\begin{aligned}
& S_{A B}(t, \rho)= \\
& = \begin{cases}S_{\max }(t)+S_{\min }(t)\left(1-S_{\max }(t)\right)(1-\rho) & \text { if } 0 \leq \rho \leq 1 \\
\left(S_{A}(t)+S_{B}(t)-S_{A}(t) S_{B}(t)\right)(1-|\rho|)+\min \left(1, S_{A}(t)+S_{B}(t)\right)|\rho| & \text { if }-1 \leq \rho \leq 0\end{cases}
\end{aligned}
$$

where $S_{\max }(t)=\max \left(S_{A}(t), S_{B}(t)\right), S_{\min }(t)=\min \left(S_{A}(t), S_{B}(t)\right)$, and $\rho$ is the correlation over all patients of the times that each patient would have survived if treated with drug A and B independently. (See Supplement for the derivation of Eq.(1).) As we vary the parameter $\rho$ in Eq.(1) using as $S_{A}(t)$ and $S_{B}(t)$ the observed PFS curves of the chemotherapy and Trastuzumab respectively, we span the range of the tCDA predictions forming the "cone" of possibilities shown in Fig. 1B, where each color corresponds to one value of correlation. 
The tCDA model embodied in Eq. (1) is based on the premise that there is a strong correlation between the survival times for each drug for a fraction $|\rho|$ of the patients, whereas the survival times for the remaining (1-| $\rho \mid)$ fraction are uncorrelated or weakly correlated (See Supplement). We can interpret this to mean that there is a sub-population consisting of a fraction $1-|\rho|$ of the patient population that can be grouped together according to some latent variable. Fig. $1 \mathrm{C}$ shows different simulations (using what we called the "Coin method" described in the Supplement) of survival times for different overall correlations in the patient population. Each point in this Figure represents a patient, and the $y$-axis and $x$-axis values are the survival times that a given patient would have survived if treated only with Trastuzumab or chemotherapy respectively. The marginal distribution of $x$-axis and $y$-axis values in all subfigures follow $S_{A}(t)$ and $S_{B}(t)$ curves respectively, and the correlations alluded to in the figure are Spearman's rank correlations. A value of Spearman's correlation equal to 1 traces the "Time Equivalence Curve" between Trastuzumab and chemotherapy (see Supplement) while a value of -1 associates the survival times of patients to drugs Trastuzumab and chemotherapy in reverse order. A Spearman's correlation of zero assigns survival times to Trastuzumab and chemotherapy independently of one another. Intermediate Spearman's correlation coefficients will have fewer points on the Spearman's correlation 1 or -1 curve a move away from order and towards disorder. From this joint distribution of patient survival times and by taking the maximum value at each ordered pair, we can construct the empirical estimate of the PFS curve under temporal CDA at a given Spearman's correlation. This simulation process is mathematically equivalent to the closed-form expression given in Eq.(1) (See Supplement and Fig. S6) which we will use throughout the paper. We will estimate the tCDA predicted PFS curve of the combination by varying the parameter $\rho$ between $[-1,1]$ in the analytical model in Eq. (1) and choosing the value of $\rho$ that minimizes the root mean square error (RMSE) between the estimated and observed combination PFS curves.

Returning to the Trastuzumab and chemotherapy trial, the optimal estimate for the Spearman's correlation is 0.03 and its corresponding $95 \%$ confidence interval is $[-0.07,0.13]$. The confidence intervals are computed via a bootstrap approach where the data is resampled many times to generate an empirical distribution of correlation estimates (See Supplement). Interestingly, a $\rho=0$, as we have almost have in this case, reduces Eq. (1) into a mathematical form analogous to that of Bliss independence used in the dose domain, namely $S_{A B}(t, \rho=0)=S_{\max }(t)+S_{\min }(t)\left(1-S_{\max }(t)\right)$. A zero Spearman's correlation indicates that the survival times that can be attributed to each of the treatments are independent of each other in the population. This suggests that in the absence of a stratification strategy to separate metastatic HER-2 overexpressing breast cancer patients into those that respond better to Trastuzumab and those that respond better to chemotherapy, it is better to administer the combination to all such patients in comparison to trying to assign each patient to the monotherapy that will work best for them. In this scenario, when we are fundamentally unable to decide for a given patient whether Trastuzumab or chemotherapy is better for them and in order to reduce the guesswork in the process, the best strategy is to give the combination for each patient. When the absolute value of the Spearman's correlation is significantly different from zero (Fig. 2A), it may be reasonable to look for confounding covariates that give rise to the observed correlation. It may be possible that these covariates could help stratify 
the patient population such that there is a subset $\rho$ of patients to whom we need not administer the combination and could only assign one of the constituent monotherapies based on a biomarker panel informed by the confounding factors. Examples of combinations for which the tCDA model yield large Spearman's correlations include 5-FU and Oxaliplatin in advanced pancreatic cancer, Interferon Alfa and Temsirolimus in advanced renal cell carcinoma, Irinotecan Bevacizumab and Panitunumab in advanced colorectal cancer, and other combination chemotherapy regimens (Figs. S3 - S5). Of course, we must be sufficiently certain that the tCDA model describes well the effect of the combination, and even in that case we must be careful to not over-interpret the meaning of the Spearman's correlation resulting from the tCDA model fit.

We can observe that the resulting estimate for the combination PFS curve under tCDA follows the true combination rather well (Fig. 1D). Empirically, using a modified Kolmogorov-Smirnov test statistic (See Supplement), we fail to reject the null hypothesis that the tCDA model sufficiently describes the observed combination ( $p$-value of 0.46 ). In this sense, it can be hypothesized that this combination is not inconsistent with the tCDA additivity mechanism.

\section{Temporal Correlated Drug Action represents a null additivity model for discerning non-additive combinations}

For 18 different combinations tested in clinical trials and retrieved from the literature (See Methods and Supplementary Table S1), we estimate the optimal tCDA predicted survival curve and assess whether tCDA is the appropriate framework to apply (Fig. 2A). Using the tCDA framework as a null model allows us to categorize clinically tested combinations as either plausible tCDA or non-tCDA via a formal hypothesis test (See Supplement). The null hypothesis is that the tCDA model adequately describes the observed combination. We use a statistical significance threshold of 0.01 to reject the null (additivity) hypothesis. In $61 \%$ of the tested combinations, we failed to rejects the tCDA model ( $p$-value $i$ 0.01) (Fig. 2A). In such cases the estimated combination PFS curve closely tracks the experimentally observed combination (Figs. 1D, 2B-F; Figs. S3C, S3D, S4A, S5A, S5C). The tCDA model can handle combinations composed of more than two drugs so long as there is some partition of the drugs within the combination into two distinct PFS curves (e.g., Fig. 2F).

Observed combinations that closely follow the best-performing monotherapy represent situations where it may be most beneficial to treat patients only with the consistently better performing monotherapy since there are fewer concerns around drug-drug interactions, toxicity, and side effects that accompany combination therapies. An example of this is the combination of Interferon Alfa and Temsirolimus in advanced renal cell carcinoma (Fig. S3C) because Temsirolimus is the consistently better performing monotherapy across time. In Fig. 2E, immediately after 15 months, the tCDA PFS estimate follows the Paclitaxel and Carboplatin monotherapy. This highlights an important characteristic of tCDA: the worstcase scenario for any combination is that the combination performs just as well as the best-performing monotherapy at a given point in time. 
The tCDA model represents a baseline model we can test for non-additive combinations. Non-additivity includes antagonism and synergy, the latter being more important for developing clinically successful combinations. $39 \%$ of the tested combinations can be classified as non-additive because we reject the null model in these cases (Fig. 2A). It is critically important to understand these non-additive combinations, as they represent the combinations that perform much better or worse than expected compared to their monotherapy results. Despite a visually good fit, we must be careful in jumping to the conclusion that tCDA quantitatively describes the effect of the actual combination. This phenomenon can be noted in the Dabrafenib and Trametinib combination in metastatic BRAF-mutant cutanous melanoma (Fig. $\mathrm{S} 5 \mathrm{~B})$. The tightness of the fit acts as a red herring, distracting us from the fact that it is in fact a non-additive interaction ( $p$-value $<0.01$ ). The non-additivity encouragingly suggests a synergistic interaction which is supported by the mechanism of action of both drugs. Trametinib is a selective inhibitor of MEK1/2 activity and Dabrafenib is a potent inhibitor of BRAF and CRAF [22, 23]. Both BRAF and MEK are in the same pathway where BRAF is upstream of MEK, so drugs that inhibit these two targets provide a classical example of synergy. There are more obviously synergistic combinations, like the 5-FU and Oxaliplatin combination for advanced pancreatic cancers for which the statistical test ( $\mathrm{p}$-value $<10^{-5}$ ) supports the visual fit and mechanistic understanding of the combination (Fig. S3A).

\section{Dose-space Correlated Drug Action is a generalized additivity model at the level of cells in culture}

In this section we extend the principle of independent drug action, originally presented to address survival of cancer patients treated with drug combinations, to the problem of determining cell viability in response to drug combinations in cell cultures and in dose space. To do so we apply the same principles that were applied for tCDA but rather than asking for the survival time of a patient under each of two drugs, we will consider the lethal dose of a cell to each of two drugs after a fixed treatment time has elapsed (typically 24,48 or 72 hours). The lethal dose, which depends on the treatment time, is a property of each cell in response to a drug. Each cell has different lethal doses for different drugs. A cell treated with a given drug at a specified dose and for a specified treatment time will be found dead (resp. alive) after that time if its lethal dose is below (resp. above) the applied dose. The extension of tCDA to cell cultures treated with a pair of drugs postulates that a cell in the culture will be viable after the treatment time has elapsed if the lethal dose of that cell for each of the monotherapies in the combination is larger than the dose of corresponding drug in the pair (see Supplement). Therefore cells that had at least one its lethal doses smaller than the applied doses in the combination will be found dead at the targeted treatment time. An important assumption in what follows is that the lethal doses of the two drugs in a combination may be correlated. The origin of this correlation could be some similarity in the mechanism of action and/or the action of some confounding variables. In cell cultures we can think that specific characteristics of a cell such as its number of mitochondria [24], its size, its protein content, etc., could be a confounder that simultaneously affect the lethal doses of each of the two drugs. We will call the application of correlated behavior of the lethal doses of cells the dose Correlated Drug Action, or 
dCDA. The dCDA model can be expressed as the following closed-form expression (see Supplement for its derivation)

$$
\begin{aligned}
V_{A B}\left(D_{A}, D_{B}, \rho\right) & =(1-|\rho|) V_{B}\left(D_{B}\right) V_{A}\left(D_{A}\right) \\
& +|\rho| \begin{cases}\min \left(V_{A}\left(D_{A}+g\left(D_{B}\right)\right), V_{B}\left(D_{B}+f\left(D_{A}\right)\right)\right) & \text { if } 0 \leq \rho \leq 1 \\
\max \left(0, V_{A}\left(D_{A}\right)+V_{B}\left(D_{B}\right)-1\right) & \text { if }-1 \leq \rho \leq 0\end{cases}
\end{aligned}
$$

where $V_{A B}\left(D_{A}, D_{B}, \rho\right)$ is the dCDA predicted viability of the cell culture for the combination of drugs $\mathrm{A}$ and $\mathrm{B}$ at doses $D_{A}$ and $D_{B}$ when the Spearman's rank correlation of the lethal doses to $\mathrm{A}$ and $\mathrm{B}$ is $\rho, V_{A}\left(D_{A}\right)$ and $V_{B}\left(D_{B}\right)$ are the dose response curves for the cell culture treated with drugs $\mathrm{A}$ and B independently. The function $f\left(D_{A}\right)$ (resp. $g\left(D_{B}\right)$ ) is the equivalent dose of A (resp. B) that would produce the same effect in the culture viability as the dose $D_{B}$ of $\mathrm{B}$ (resp., the dose $D_{A}$ of $\mathrm{A}$ ) did, and are discussed in the Supplement.

The dCDA framework accommodates a spectrum of additive models previously reported in the literature (see Supplement). For $\rho=0$ the dCDA model reduces to the Bliss additivity model. For $\rho=1$ the $\mathrm{dCDA}$ model reduces to a modified version of the HSA model that is sham compliant. sham compliance should be expected when $\rho=1$. Indeed, when a drug is 'combined' with itself the lethal doses of a cell to the same drug would result in a Spearman's correlation value of $\rho=1$.

The dCDA model requires that we find the optimal parameter $\rho$ across a range of different doses in order to estimate the combination viability in the dCDA framework. To do that we iterate through all possible values of $\rho$ in small step sizes and choose the $\rho$ that yields the combination estimate with minimum root mean square deviation from the observed viabilities (See Supplement). Across the 26 experimentally tested combinations discussed below (Supp. File 2), the average Spearman's correlation estimate is 0.03 and ranges from -0.14 to 0.21 (Fig. 3A). For a given combination estimate, we assess whether the viability of a combination predicted by the dCDA model sufficiently describes the observed viability of the combination using a two-sample paired t-test to achieve (Figs. 3D, F; See Supplement). We chose this method to minimize false negative rates, even when it results in some extra false positive CDA abiding combinations, for which we should have rejected the CDA null hypothesis. To compensate for this increase in false positive combinations, we will introduce later on a "local analysis" that flags specific doses of combinations as non abiding with the CDA model.

To understand the extent to which the dCDA model captures the viability of cells in culture, we performed 26 sets of viability experiments under different doses and treatment times (see Methods). $80.7 \%$ of the combinations we studied were reasonable describable under the dCDA model (Fig. 3A X-labeled bars) of which a subset was also describable under the Bliss additivity model (Fig. 3A double-dagger-labeled bars). The latter is simply the dCDA model for $\rho=0$. Equivalently, we can compute the difference between the estimated combination viability under the dCDA model and the observed combination viability, a metric we term the Excess over CDA (EOCDA). EOCDA is a measure of non-additivity, and can be visualized as a heatmap where negative values (more blue) indicate an underestimation of the 
true viability and positive values (more red) indicate an overestimate of the true viability (Fig. 3C, E). If a combination is describable under dCDA, then the EOCDA metric also holds as an appropriate measure (Fig. 3B green and blue dots).

Classifying combinations as describable by the dCDA model or not is a global characterization and represents a measure across all of the combination viabilities taken together. We can be more granular and attempt to understand which specific doses in a dCDA compliant combination are non-additive. Let us first consider Fig. 3D and $F$ where each $(x, y)$ coordinate represents the (estimate, true) combination viability and are colored according to the EOCDA color scale. Possible antagonistic drug doses in this setup will be bluer points and possible synergistic drug doses will be redder points. Through a leaveone-out cross validation re-estimation framework (See Supplement), we can assign each specific point (corresponding to specific doses of the two monotherapies) as locally additive or non-additive ( $p$-value threshold of 0.01 for minimizing rate of falsely labelling points as non-additive). For instance, in Fig. 3D none of the points are locally non-additive but in Fig. 3F there are two such points (both likely synergistic). Of particular note, for the cases globally describable under dCDA presented in Fig. 3C, E and in igs. S10 - S17, there are at most a few doses per combination that display locally non-additive behavior.

It is plausible, but not necessary, that the Spearman's correlation estimate in the dCDA model describes to some extent the similarity between mechanisms of action of the individual drugs that constitute the combination. If that were the case, the larger the absolute value of the Spearman's correlation estimate, the more similar the mechanisms of action are expected to be. Since our Spearman's correlation estimates are on average not far from zero, we would expect that combinations describable under dCDA (GoF p-value $>0.01$ ) are composed of monotherapies whose mechanisms of action are effectively unrelated. For example, Tamoxifen targets the estrogen receptor in breast cancers whereas GW 8510 is a cyclin-dependent kinase inhibitor investigated with respect to colorectal cancer $[25,26]$. These two mechanisms of action are not related in any intuitive manner. This lack of a relationship is represented by a Spearman's correlation estimate close to zero.

The majority of the tested combinations we studied were assayed at 24 hours after administering the drugs, but a select few were collected in a time-series experiment. There are two sets of combinations for which time-series viability data was collected. Experiments 21, 22, 23 correspond to Tamoxifen and Withaferin A at 12, 24, and 48 hours respectively (Fig. S17). The Spearman's correlation estimate was stable over time but the EOCDA p-value decreased over time $(0.68,0.15,3.3 e-5)$. Thus, the dose-space CDA additivity model sufficiently described the combination until the 48 hour time point. Experiments 5, 11, 26 correspond to Tamoxifen and Mefloquine at 12, 48, and 24 hours respectively (Fig. S16). The Spearman's correlation estimates varied over time $(0.12,-0.05,0.02)$ but the EOCDA p-values were consistently above the threshold for rejection of the dose-space null additivity model ( $p$-value $>0.01$ ). From both time-series experiments, we can observe that there may be undiscovered temporal dynamics at play with respect to the action of drug combinations in cell culture. 
Independent of the aforementioned six experiments, we collected data for Tamoxifen and Mefloquine at 24 hours in Experiment 14 and data for Tamoxifen and Withaferin A at 24 hours in Experiment 12. As part of the 20 non-time series trials, we didn't collect pure monotherapy viabilities but rather the viability at which the other drug in the combination was given in the lowest tested dosage. Thus, we can assess the robustness of the model to non-ideal input data by comparing Experiment 14 to Experiment 26 and Experiment 12 to Experiment 22. The Spearman's estimate differs by 0.05 between Trial 14 and 26 and in both cases the dCDA model sufficiently describes the true data (Fig. 3A). The Spearman's estimate differs by 0.01 between Trial 6 and 25 and once again, the dose-space CDA model p-value is concordant between trials (Fig. 3A). These results favorably suggest that non-ideal input data does not considerably affect the performance of the model.

Excess over Bliss (EOB), one of the most popular performance metrics for assessing the synergistic or antagonistic behavior of a combination at particular dosages, is based on Bliss independence. In order for EOB to be a valid measure, the underlying combination must be describable under Bliss independence. Given that the dose-space CDA model collapses to Bliss independence when $\rho=0$, we can formally test whether the combination can be sufficiently explained by Bliss independence (See Methods). The EOB condition is valid for $42.3 \%, 11$ of the 26 , of tested combinations (Fig. 3A, double-dagger-labelled bars). Intuitively, the closer the optimal Spearman's correlation estimate is to zero, the more likely the $E O B$ condition is to be valid. We can also investigate the joint distribution of p-value statistics for the EOB and EOCDA status of each tested combination (Fig. 3B). Most of the combinations tested can be described by dCDA (blue and green dots in Fig. 3B) whereas much fewer can be described by Bliss independence (blue and cyan dots in Fig. 3B). At the intersection, 38.5\% of combinations are both describable under dCDA and satisfy Bliss independence (Fig. 3B, blue dots). It is important to note that, on the whole, the combinations describable under Bliss are a subset of those describable under dCDA. This highlights the strength of dCDA and EOCDA - we minimize our chances of identifying a combination as non-additive when it in fact is additive.

\section{Discussion}

We introduced Correlated Drug Action (CDA) as a baseline model of additivity in the temporal and dose domains. Specifically, we discussed the tCDA model for survival times in patient populations and the dCDA model for cell viabilitiy in cell cultures. Both models can be formally interpreted as baseline models of additivity in their respective domains. We showed how these baseline models can be considered as the null hypothesis of a statistical test that allows us to reject CDA as the additivity model for sufficiently low p-value. Assessing non-additivity is important to better understand combination therapies and to narrow down which combinations to investigate further.

As a model of survival times in patient populations, tCDA builds upon Palmer and Sorger's independent drug action (IDA) approach which assumes that the two drugs in a combination do not directly interact 
[7]. As in IDA, tCDA also assumes that the times that each patient would survive under each of a pair of monotherapies if delivered independently are correlated in the population. There are many ways in which this correlation can be modeled. In order to test if different correlation models lead to similar or different results, we developed two Monte Carlo simulation methods (the "coin" and "window swap" methods; see Supplement) to compare the effect that similarly correlated but with different underlying joint distribution of survival times would have in the combination. The results of both simulation methods are highly concordant on simulated monotherapy survival distributions (Figs. S7A and B) and, for the most part, on the real monotherapy survival distributions (Figs. S7C,D). Given the concordance between these two quite distinct methods, we chose the coin method to be the basis for tCDA framework as it allows us to express the model using a closed-form mathematical expression (see Supplement, and Fig. S6). Overall, these results suggest that although there are many ways to create survival time pairs with a given correlation, the tCDA model captures the survival curve characteristics of different methods such as the coin method (Fig. S8) and the window-swap method (Fig. S9). In addition, the tCDA model offers a computational advantage in terms of efficiency at the time of fitting the model to the data, given that we only need to use a formula rather than simulations to find the correlation. Additionally, we endowed the tCDA model with a hypothesis testing procedure to reject the tCDA additivity model as the null hypothesis. The tCDA model can describe remarkably well a subset of the clinically relevant combination cancer therapies (Fig. 1,2A) studied here. The public data collected in this work to validate the tCDA model uses data from 18 clinical trials, which include nine that were described in [7].

In our hypothesis testing framework, we identify the Nivolumab and Ipilumumab combination in untreated melanoma (Fig. 2D) as an additive combination with a 95\% confidence interval for $\rho$ spanning 0.275 to 0.445 . Our results for this trial are concordant with the simulation estimate of 0.25 computed in [7]. This trial is a key immunotherapy regimen with much clinical relevance. Our results suggest that the progression-free survival benefits of this combination can be attributable to correlated drug action. In our framework and the related Sorger model, patient to patient heterogeneity is an important factor in determining population-level response to a therapy. Our work accounts for this heterogeneity, at least in part, through the estimated parameter $\rho$. Taking the tCDA model literally, the parameter $|\rho|$ represents the fraction of patients that lie on the $\rho=1$ line (Fig. S6B), that is, whose survival time to one drug determines the survival time to the other drug. This suggests that for a given combination, there may be some set of possibly undiscovered latent variables that can be used to identify a fraction $|\rho|$ of patients for whom the survival times for both monotherapies are related. On the other hand, there is a fraction $1-|\rho|$ of patients whose survival times to each of the monotherapies in the combination are truly independent. For combinations that are sufficiently describable with the tCDA model with a moderate to high absolute value of the Spearman's correlation (Fig. 2F; Figs. S3C, S4A, S5A), the previous discussion suggests that there may be a stratification of patients such that we need only to give them the drug with better survival for them. Contrarily, when the Spearman's correlation is near zero (Figs. 1, 2C, Fig. S3D), it suggests that there is no common latent variable affecting the action of both monotherapies. Under this scenario, we recognize that the combination has maximal benefit since there 
is no relationship between the two monotherapies' survival times. Most importantly, in these situations, we are able to leverage our uncertainty by giving the entire combination to each patient. More work is necessary to validate this conjecture, which follows from a literal interpretation of the tCDA model.

In order to collect clinical trial data that was in the format necessary for the tCDA model, we often had to aggregate data from up to three different clinical trials (Supp. Table 3). In these scenarios, we attempted to match cohort characteristics including sex, age, ethnicity, and previous treatment history as well as possible.

The tCDA model, which provides a baseline additive survival probability for drug combinations in patient populations, can be extended to a baseline additive model for the viability of cells in cell cultures at different doses, which we called the dCDA model. A key feature in the theory leading to the dCDA model is the assumption that for each cell in culture subjected to a drug for a certain treatment time $T$, there exists a lethal dose (dependent on $T$ and varying from cell-to-cell) such that, if the drug is applied at a dose lower (resp. higher) than the lethal dose, the cell will be found alive (resp. dead) after the given time $T$. If two drugs are applied for a time $T$ in combination and at their respective doses, the dCDA model postulates that only those cells for which both lethal doses are higher than the doses at which the drugs in the combination were applied will survive. Note that the lethal dose for each cell in a culture in the dCDA context plays a similar role to the survival time for each patient in a population in the tCDA context. Like the tCDA model, the dCDA model can be formulated as a closed-form expression under the assumption of a particular joint distribution of lethal doses in the cell population, parameterized by the Spearman's correlation $\rho$ between lethal doses. There have been many proposed additivity models for the viability of a cell culture to a drug combination, including the Bliss additivity model and the Highest Single Agent (HSA) model, as well as several schemes to treat dose effects such as the Dose Equivalence Principle. The dCDA model reduces to the the Bliss additivity model for $\rho=0$ and, by leveraging the Dose Equivalence Principle, to a sham-combination compliant version of the HSA model for $\rho=1$ (See Supplement). This defines a spectrum of models wherein varying the Spearman's correlation from zero to one controls whether the combination is more Bliss-like or more sham compliant HSA-like. The actual value of $\rho$ is determined by the data, and estimated by our framework (Fig. 3 and Supp. Table 2). Thus our paradigm combines the advantages of several ways of thinking about additivity.

By providing an integrated baseline model of additivity it is possible to determine if a drug combination is synergistic or antagonistic. In order to do that, we introduced a new metric which we called Excess over Correlated Dose Action, or EOCDA, which is simply the difference between the viability estimated using the dCDA model and the observed viability; positive EOCDA values correspond to synergistic combinations and negative EOCDA values correspond to antagonistic combinations (Fig. 3C,E). We compared the EOCDA metric to the popular Excess over Bliss (EOB) metric. For both the EOCDA and $E O B$ metrics we defined statistical significance test to determine if the null hypothesis of additivity $(E O C D A=0$ or $E O B=0)$ should be rejected. Therefore, for each of the tested combinations we have a $\mathrm{p}$-value for the null hypothesis $\mathrm{EOB}=0$ and $\mathrm{EOCDA}=0$. Fig. $3 \mathrm{~B}$ illustrates the degree of relatedness 
between the two metrics. We can notice that there are more cases for we cannot reject the null hypothesis of additivity (at a significance level of 0.01 ) under the dCDA compared to Bliss additivity (more blue and green dots than blue and yellow dots in Fig. 3B). In other words, EOCDA reduces the chance of calling a combination non-additive compared to EOB - an important criteria when considering the experimental resources and effort that it takes to validate non-additivity. One limitation of EOCDA, however, is that we need the entire dose matrix with corresponsing viabilities to estimate the parameter $\rho$ whereas $\mathrm{EOB}$ can be computed at individual dosages. Interestingly, for combinations describable under dCDA, we observe that there are only a few doses that are "locally" non-additive in a globally additive combination. This plausibly identifies dosages of particular interest for further investigation.

As discussed earlier, the fitted parameter $\rho$ in the dose-space model corresponds to the Spearman's rank correlation between the lethal doses of two different therapies for one cell across the cell culture. The Spearman's correlation provides us information regarding the association between the two monotherapies including the possibility of the two drugs have similar mechanisms of action. The reason we are able to discuss possible biological explanation from a much more general definition in a cell culture case is that unlike patients, cell cultures are relatively homogeneous and isogenic tumor cells free of the external milieu and confounders that exists in a human population. In our data, all of the correlation estimates are weak implying that, from the perspective of dCDA, the drugs probably act using alternative pathways (Fig. 3A). For example, GW 8510 is a cyclin-dependent kinase inhibitor whereas Withaferin A inhibits Vimentin, an intermediate filament. These two mechanisms are not linked by any known direct relationship, and this supports the low Spearman's correlation estimate given by the dose-space CDA model (Fig. 3E, F). More work is needed to validate the notion that a similarity in the mechanism of action of two drugs can be reflected in the value of the parameter $\rho$.

There are many possible avenues for extending this work. It seems plausible that the CDA framework can be extended to combination approaches outside the field of cancer including HIV/AIDS, antibiotic resistant infections, malaria, heart disease, and other clinical conditions. In each case the appropriate disease-relevant metric should be chosen as the right endpoint, such as time to response, or time to resistance. Another line of work to extend this work could be the creation of a network of therapies for a given cancer. Each node is a particular monotherapy and the edge width between two nodes corresponds to the Spearman's correlation estimate. This could be done for cell cultures or patient populations. From this setup, we can attempt to learn on the graph and infer the edge strength between other pairs of nodes. This could be informative in suggesting new candidates for cell line experiments or clinical trials. Additionally, we can investigate the relationship between the dose-space and temporal CDA models by testing the same cancer combination therapy in mice PDX models and patient cancer cells cultured in the lab. 


\section{Methods}

\section{Collection of clinical trial data}

We collected data from clinical trials using the clinicaltrials.gov query tool and scraped Progression Free Survival (PFS) data from their associated results and papers. We converted graphs into tables of values through a robust online digitizer (link). Though it would be best to have the original data, these files were not available to us. For a given combination, we aimed to find a single trial that tested the individual therapies and the combination. However, we also had to pull data across different trials to construct the necessary survival data. In these cases, we tried to maximize overlap in patient characteristics, dosages, and time schedules. We were able to match dosages and time schedules in the overwhelming majority of cases. We assembled data for 18 distinct combinations spanning 10 different cancer types and a detailed guide to the raw data can be found in Supp. Table 3. The tables of the raw PFS curves from the digitized plots that we used for our analysis can be found in Supp. File 1.

\section{tCDA model}

Code for the Temporal CDA Model, tCDA, can be found here. Results for tCDA on the clinical trials can be found in Supp. Table 1.

\section{Drug Combination Experiments in the MCF7 cell line}

\section{Choice of Drug Combinations, Dose matrix and Treatment Times}

We applied the dCDA model to fit the viability of MCF7 cells in response to 26 drug combinations. These 26 drug combinations comprised 20 unique combinations, and 6 repeat combinations as discussed below. The 20 combinations, which included 13 unique drugs (Atovaquone, Emetine, GW 8510, Imatinib, Mefloquine, MG 132, Mitoxanthrone, Nicardipine, Sanguinarine, Tamoxifen, Terfenadine, Tyrphostin AG 825 and Withaferin A), were selected from a a pre-existing drug combination screen collected at Columbia University (Andrea Califano lab) in the context of the Library of Integrated Network-Based Cellular Signatures (LINCS) Program. This screen tested all 990 combinations of 99 drugs against 10 drugs, each combination assessed in a dose response matrix of $4 \times 4$ doses. Of these combinations we chose a subset of combinations that exhibited antagonistic, additive and synergistic behaviors, resulting in the 20 combinations reported here. The 20 drug combinations were administered at doses shown in the $6 \times 6$ dose matrices shown in Figs. 3, and Figs. S10 - S15, and the viability was measured at 24 hours. These 20 experiments, which we refer to as the $24 \mathrm{~h}$ viability experiments, are reported for the first time in this paper and provided as Supp. File 2. Amongst the 20 drug pairs, two specific combinations (Tamoxifen/Withaferin A and Tamoxifen/Mefloquine) were measured at more detailed dose matrices and treatment times as reported in an earlier publication [27]. For these two pairs, 
viability was measured at the doses corresponding to the $10 \times 10$ dose matrix shown in Figs. S16 and $\mathrm{S} 17$, and at treatment times of $12 \mathrm{~h}, 24 \mathrm{~h}$ and $48 \mathrm{~h}$. We also measured the viability of the individual monotherapies (Tamoxifen and Mefloquine) at the 10 doses reported in the figures, at $12 \mathrm{~h}, 24 \mathrm{~h}$, and 48h. These 6 experiments, which we will refer to as the time course viability experiments, had been previously made publicly available in [27]; we also provide these data for convenience in Supp. File 2. All drug combination viability experiments (the $24 \mathrm{~h}$ and time course experiments) were done in triplicate and the results for each combination were averaged. All monotherapy experiments (for the time course data) were done in duplicate and the results averaged. The dCDA model requires that we have viability for the monotherapies. We had that data for the time course data, but we lacked the equivalent data for the $24 \mathrm{~h}$ viability experiments. Therefore to apply the dCDA model, we approximated the cell viability under individual drugs by considering the case when the other drug in the combination was measured at its lowest concentration.

Cell Culture

MCF7 (ATCC HTB-22) cells were obtained from ATCC. Cells were cultured according to manufacturer's recommendations in ATCC-formulated Eagle's Minimum Essential Medium (Catalog No. 30-2003) with $10 \%$ heat-inactivated fetal bovine serum, and $0.01 \mathrm{mg} / \mathrm{ml}$ human recombinant insulin. Growth media was changed every 3-4 days. After reaching confluence, cells were split at a ratio 1:6. Cultures were tested for mycoplasma periodically using MycoAlert (Lonza, Cat No. LT07-701) per manufacturer's instructions. To split, media was removed, cells were washed with PBS, and trypsin-EDTA mix was added for $5 \mathrm{~min}$. After detachment, cells were washed with growth media, collected into $50 \mathrm{ml}$ vial, spin down at 1000 RPM, suspended in fresh media and plated into $75 \mathrm{~cm}$ flasks. The cells were plated at 10,000 cells per well in a clear bottom black 96-well plate (Greiner Cat. No. 655090) and a white 96-well plate (Greiner Cat. No. 655083) then they were placed in an incubator. After $24 \mathrm{hr}$, the plates were removed from the incubator and treated with drugs using the HP D300 Digital Dispenser.

\section{Cell viability}

Cells were then treated with the appropriate drug combinations at specific dose matrices and treatment times. After the targeted drug treatment time, $100 \mu \mathrm{L}$ of Cell-Titer-Glo (Promega Corp.) was added to the wells in the white 96 -well plate and shaken at $500 \mathrm{rpm}$ for $5 \mathrm{~min}$. The plate was then read by the Perkin Elmer Envision 2104 using an enhanced luminescence protocol to count the number of raw luminescent units per well. For each viability experiment, the results were normalized by the luminescence measured in untreated cell cultures. The ratio of luminescence in treated vs untreated cells is what we report as cell viability.

\section{dCDA model}

Code the dose-space CDA model, dCDA, can be found here here. Results for dCDA on the experimental combinations can be found in Supp. Table 2. 


\section{Acknowledgements}

We thank the Columbia University High Throughput Screening Facility and the Sulzberger Columbia Genome Center, where the viability experiments were conducted by Charles Karan and Ronald B. Realubit. We also thank Andrea Califano for providing the LINCS screen of 990 drug combinations from where we chose the drug combinations for cell viability used in this paper.

\section{Authors' contributions}

GS conceived of the project. All co-authors developed the methodology for the project. ASA and SCK developed computational approaches and performed bioinformatics analysis with guidance from GS and MEA. ASA drafted the manuscript and all authors participated in its editing and revision.

\section{Funding}

Funding for this work was provided by IBM Research and the Icahn School of Medicine at Mount Sinai (ISMMS).

\section{Competing interests}

The authors declare that they have no competing interests.

\section{Availability of Data and Materials}

All data and code can be found at github.com/aditharun/correlated-drug-action.

\section{References}

[1] Ann E. Kurth et al. Combination HIV Prevention: Significance, Challenges, and Opportunities. Curr HIV/AIDS Rep., 8(1):450-470, 2011.

[2] Emil Frei III et al. Studies of Sequential and Combination Antimetabolite Therapy in Acute Leukemia: 6-Mercaptopurine and Methotrexate. Blood, 18(4):431-454, 1961.

[3] Emil J. Freireich et al. The Effect of 6-Mercaptopurine on the Duration of Steroid-induced Remissions in Acute Leukemia: A Model for Evaluation of Other Potentially Useful Therapy. Blood, 21(6):699-716, 1963. 
[4] Reza Bayat Mokhtari, Tina S Homayouni, Narges Baluch, Evgeniya Morgatskaya, Sushil Kumar, Bikul Das, and Herman Yeger. Combination therapy in combating cancer. Oncotarget, 8(23):38022, 2017.

[5] Emil Frei III et al. The Effectiveness of Combinations of Antileukemic Agents in Inducing and Maintaining Remission in Children with Acute Leukemia. Blood, 26(5):643-656, 1965.

[6] Jonathan B. Fitzgerald et al. Systems Biology and Combination Therapy in the Quest for Clinical Efficacy. Nature Chemical Biology, 2(9):450-470, 2006.

[7] Adam C. Palmer and Peter K. Sorger. Combination Cancer Therapy Can Confer Benefit via Patient-to-Patient Variability without Drug Additivity or Synergy. Cell, 171(7):1678-1691, 2017.

[8] William R Greco. The search for synergy: a critical review from a response surface perspective. Pharmacol Rev, 47:331-385, 1995.

[9] CT Meyer, DJ Wooten, BB Paudel, J Bauer, KN Hardeman, D Westover, et al. Quantifying drug combination synergy along potency and efficacy axes. cell syst. 2019; 8: 97-108. e16.

[10] Mark Sinzger, Jakob Vanhoefer, Carolin Loos, and Jan Hasenauer. Comparison of null models for combination drug therapy reveals hand model as biochemically most plausible. Scientific reports, $9(1): 1-15,2019$.

[11] Cong Chen, Fang Liu, Yixin Ren, Leah Suttner, Zhiping Sun, Yue Shentu, and Emmett V Schmidt. Independent drug action and its statistical implications for development of combination therapies. Contemporary Clinical Trials, 98:106126, 2020.

[12] C.I. Bliss. The Calculation of Microbial Assays. Bacteriol Rev., 20(4):243-258, 1956.

[13] S Loewe. The Problem of Synergism and Antagonism of Combined Drugs. Arzneimittelforschung, 3(6):285-290, 1953.

[14] Julie Foucquier and Mickael Guedj. Analysis of drug combinations: current methodological landscape. Pharmacology research \& perspectives, 3(3):e00149, 2015.

[15] M.C. Berenbaum. What is Synergy? Pharmacol Rev., 41(3):422, 1989.

[16] Vincent T. DeVita Jr and Edward Chu. A History of Cancer Chemotherapy. Cancer Res., 68(21):8643-8653, 2008.

[17] Justin R. Pritchard et al. Understanding Resistance to Combination Chemotherapy. Drug Resist Updat., 15(0):249-257, 2012.

[18] Vincent T. DeVita Jr and Steven A. Rosenberg. Two Hundred Years of Cancer Research. N Eng/ J Med., 266(23):2207-2214, 2012. 
[19] George P. Canellos et al. Chemotherapy of Advanced Hodgkin's Disease with MOPP, ABVD, or MOPP Alternating with ABVD. N Engl J Med, 327(21):1478-1484, 1992.

[20] Jing Tang et al. What is Synergy? The Saariselkä agreement revisted. Front Pharmacol., 6(181), 2015.

[21] D.J Slamon et al. Use of chemotherapy plus a monoclonal antibody against HER2 for metastatic breast cancer that overexpresses HER2. N Engl J Med., 344(11):783-792, 2001.

[22] Iwona Lugowska et al. Trametinib: a MEK Inhibitor for Management of Metastatic Melanoma. Onco. Targets Ther., 8(1):2251-2259, 2015.

[23] Samantha Bowyer et al. Dabrafenib and Its Use in the Treatment of Metastatic Melanoma. Melanoma Manag., 2(3):199-208, 2015.

[24] Luís C Santos, Robert Vogel, Jerry E Chipuk, Marc R Birtwistle, Gustavo Stolovitzky, and Pablo Meyer. Mitochondrial origins of fractional control in regulated cell death. Nature communications, 10(1):1-10, 2019.

[25] M.M. Gottardis and V.C. Jordan. Antitumor Actions of Keoxifene and Tamoxifen in the Nnitrosomethylurea-induced Rat Mammary Carcinoma Model. Cancer Res., 47(15):4020-4024, 1987.

[26] Y-Y Hsieh et al. Repositioning of a Cyclin-Dependent Kinase Inhibitor GW8510 as a Ribonucleotide Reductase M2 Inhibitor to Treat Human Colorectal Cancer. Cell Death Discov., 2, 2016.

[27] J.E. Diaz et al. The Transcriptomic Response of Cells to a Drug Combination is More Than The Sum of the Responses to the Monotherapies. eLife, 9, 2020. 


\section{Figures}
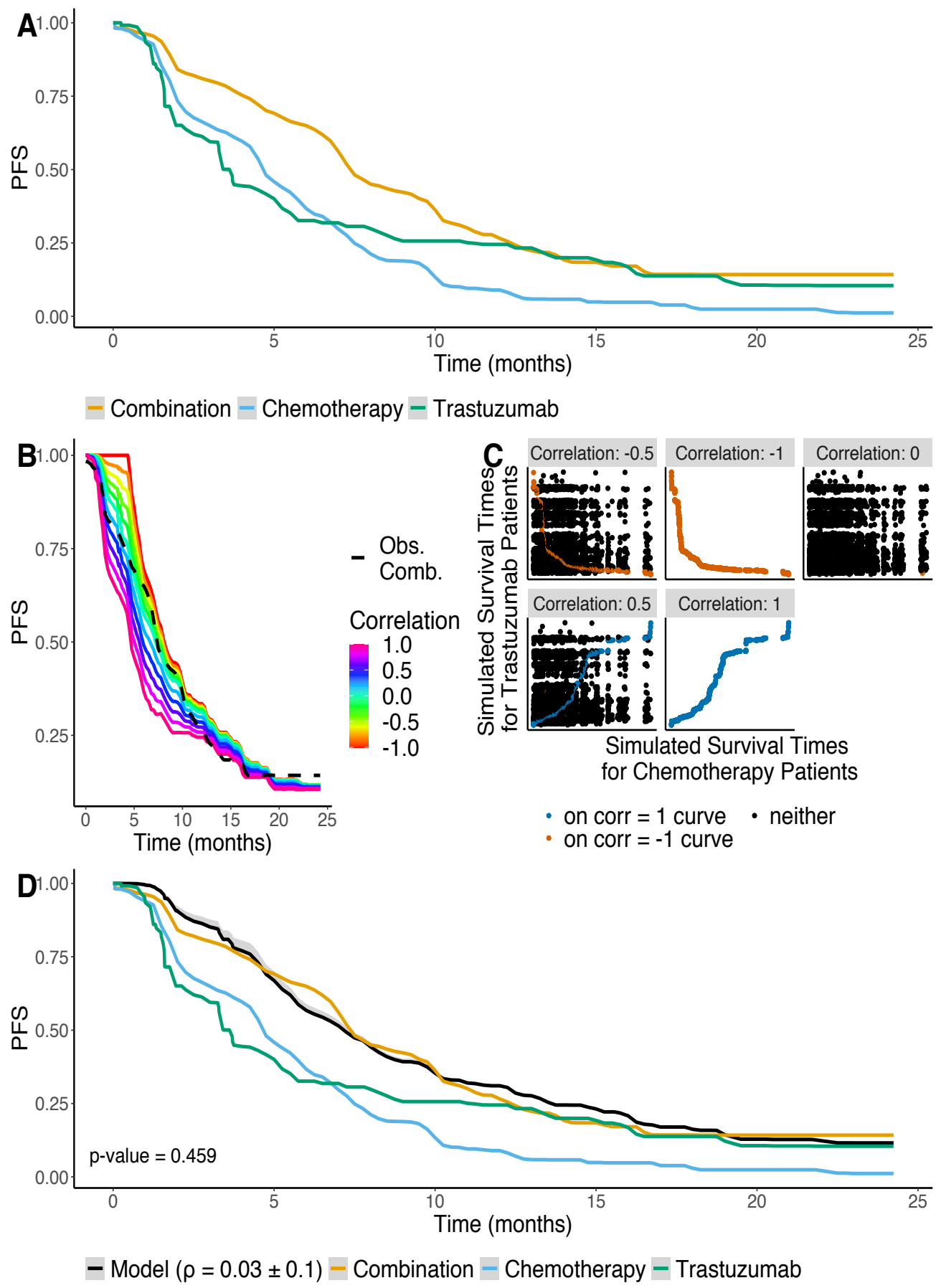

Figure 1: Correlated Drug Action explains the benefit of Trastuzumab and Chemotherapy in Metastatic HER-2 Overexpressing Breast Cancer. A) Progression Free Survival (PFS) as a function of time for either individual therapy option (Trastuzumab - green, Chemotherapy - blue) and their combination (yellow) in patients with HER-2 overexpressing breast cancer. B) Range of possible survival curves for the combination under tCDA. The observed combination (black) falls within the lines of the predicted field. C) For a given Spearman's correlation, each point represents a pair of possible PFS times associated with each simulated patient, and the parallel maximum of this paired vector of PFS times defines the combination PFS curve shown in $\mathbf{B}$. The absolute value of the Spearman's correlation corresponds to the fraction of points that lie on the correlation -1 or 1 curves (Fig. S6). D) Estimate of the combination under the tCDA model (black) and in grey are the PFS curves for the $95 \%$ confidence interval of $\rho_{s}(0.03 \pm 0.1, \mathrm{p}$-value $=0.459)$. This suggests that the tCDA additivity model sufficiently describes the effect of the combination. 

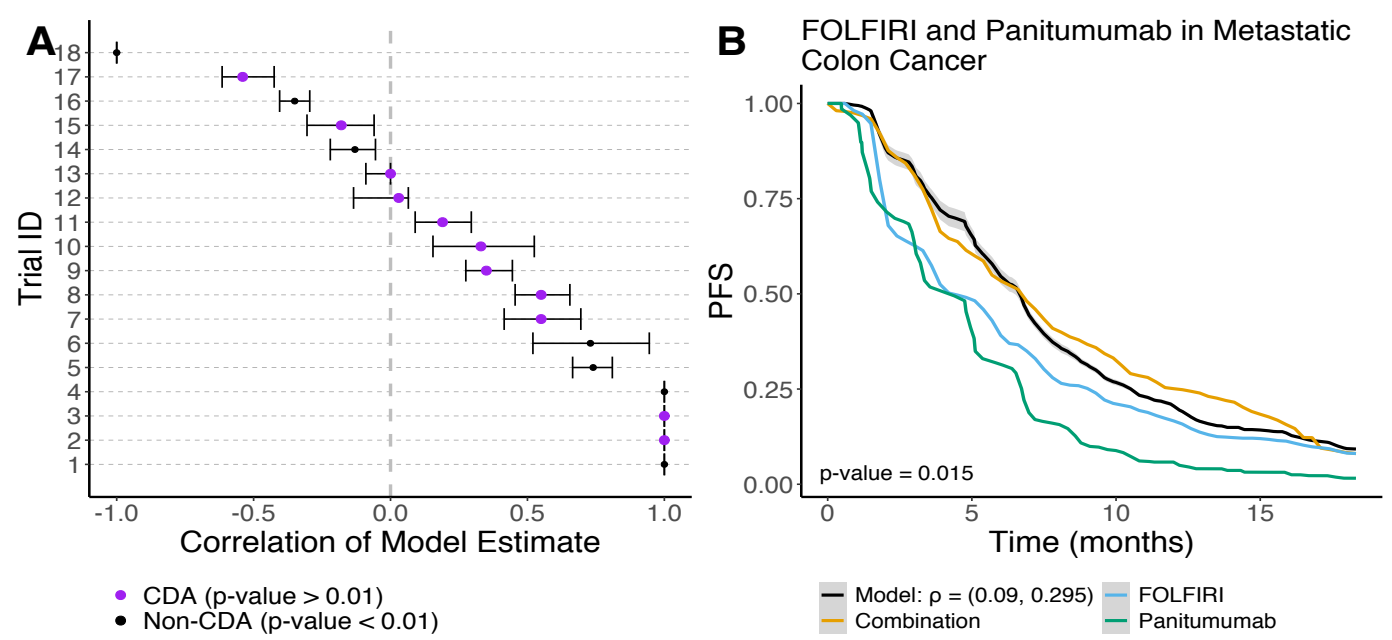

C Vinflunine and Capecitabine in Advanced

D Nivolumab and Ipilimumab in Untreated
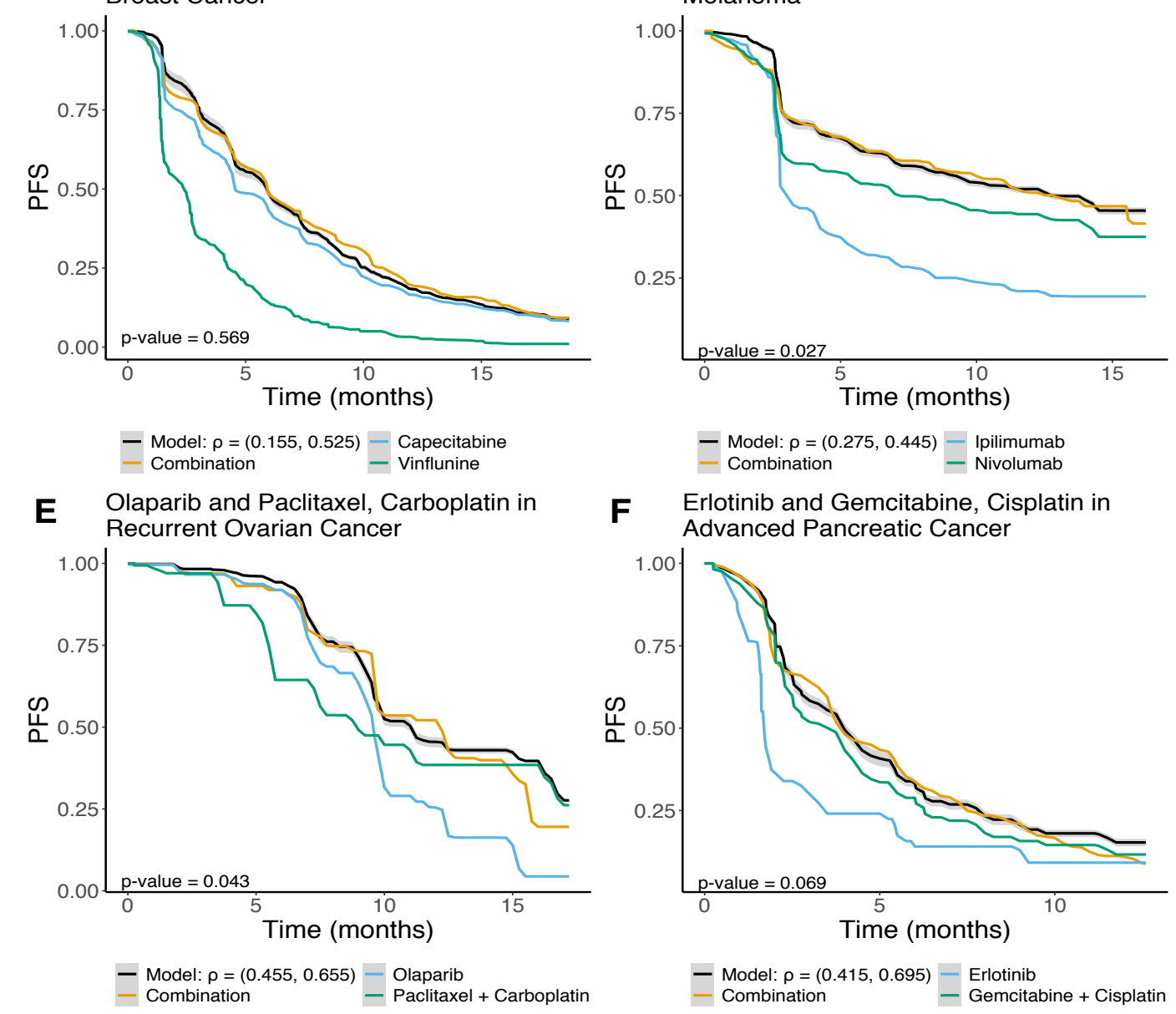

F Erlotinib and Gemcitabine, Cisplatin in F Advanced Pancreatic Cancer

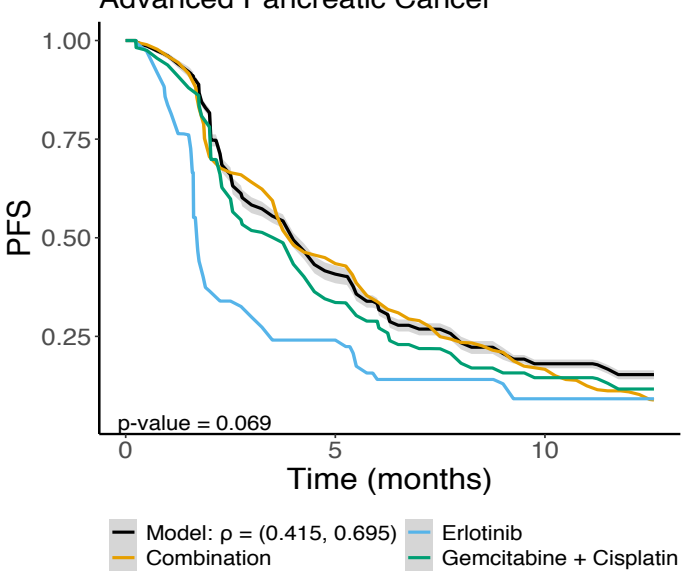

Figure 2: Temporal Correlated Drug Action (tCDA) model results. A) Optimal Spearman's correlation and associated $95 \%$ confidence interval. Purple represents trials where the effect of the combination can be explained sufficiently well under the tCDA model. The trial ID's reference the tested clinical trials, and a lookup table can be found in Supp. Table 1. B) tCDA model estimate for the combination of FOLFIRI and Panitumumab in metastatic colon cancer. C) tCDA model estimate for the combination of Vinflunine and Capecitabine in advanced breast cancer. D) tCDA model estimate for the combination of Nivolumab and Ipilumumab in previously untreated melanoma. E) tCDA model estimate for the combination of Olaparib and Paclitaxel, Carboplatin in recurrent ovarian cancer. F) tCDA model estimate for the combination of Erlotinib and Gemcitabine, Cisplatin in advanced pancreatic cancer. 

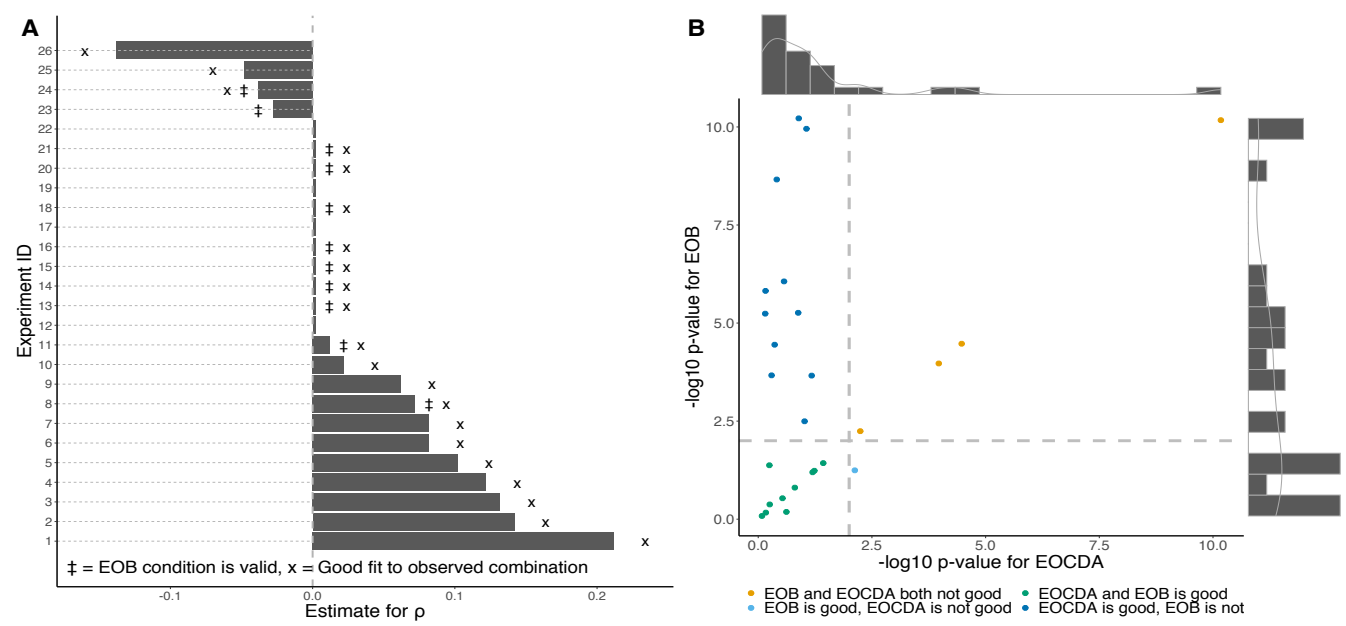

Emetine and Imatinib Combination
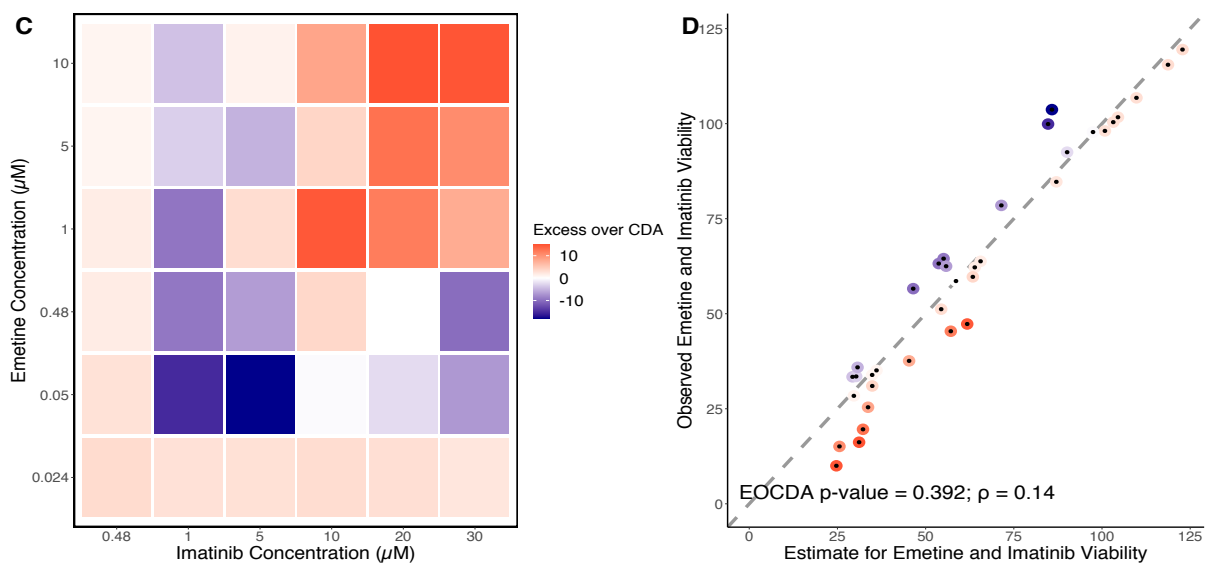

GW 8510 and Withaferin A Combination
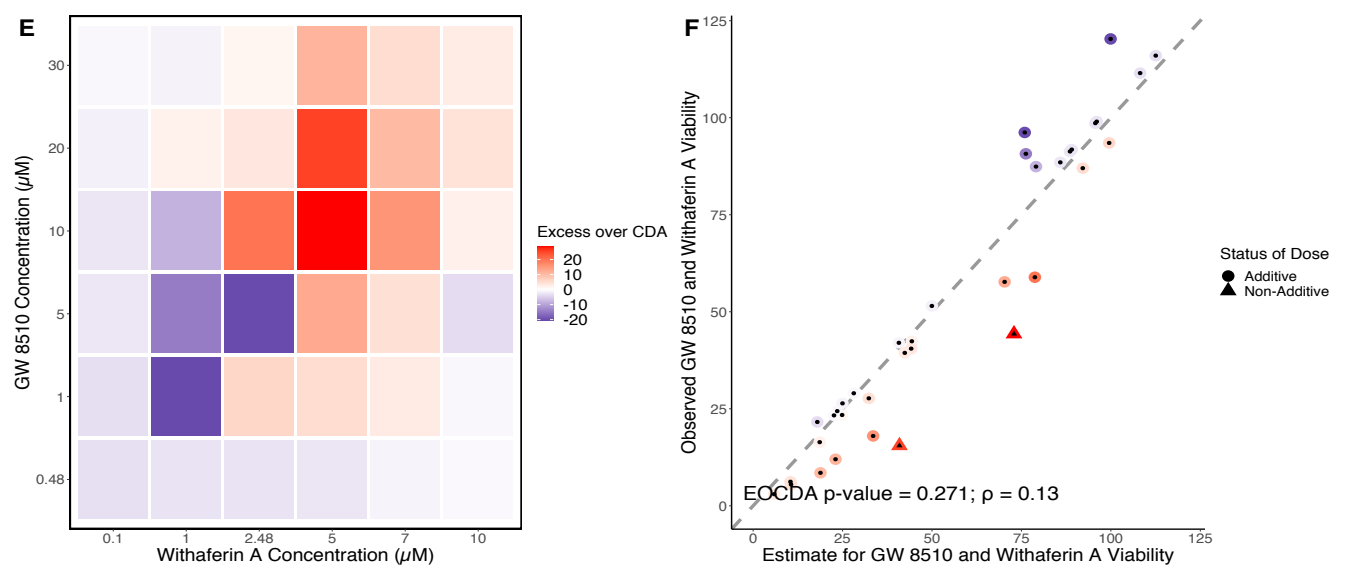

Figure 3: Dose-space Correlated Drug Action (dCDA) model results. A) Optimal Spearman's correlation estimate for each in vitro drug combination tested on MCF7 cells under the dCDA model. Double-dagger indicates a good fit ( $p$-value $>0.01$ ), and star indicates that Excess Over Bliss (EOB) is a valid metric ( $p$-value $>0.01)$. The trial ID's correspond to a given drug combination (Supp. Table 2). B) Joint distribution of p-value statistics for Goodness of Fit and EOB conditions (Dashed lines = p-value of 0.01). C, D) Emetine and Imatinib combination results. C) Heatmap of the EOCDA, predicted minus observed combination viabilities, metric. Positive values (more red) suggest synergy whereas negative value suggest antagonism (more blue). D) Comparison of estimated and observed viabilities of Emetine and Imatinib in reference to the identity line ( $p$-value $=0.392$ ). E, F) GW 8510 and Withaferin A combination results. E) Heatmap of EOCDA. F) Comparison of estimated and observed viabilities ( $p$-value $=0.27$ ). $\mathbf{D}, \mathbf{F})$ Points are colored with the same scale as its corresponding EOCDA matrix. If the GoF p-value $>0.01$ for the overall combination, then each point (i.e., dose) is classified as additive or non-additive (See Supplement). 